\title{
Ovarian activity in beef and dairy cows with prolonged postpartum period and heifers that fail to conceive
}

\begin{abstract}
The primary objectives of this study were to investigate incidence of abnormal ovarian cyclicity (AOC) and its type in dairy and beef cows with prolonged postpartum period (>90 days) and in heifers that fail to conceive. A total of 53 animals were included in the study: 17 Friesian crosses, 16 Braford crosses, eight Brangus crosses, and 12 local Kedah-Kelantan (KKX) crosses. These animals were initially checked for absence of pregnancy via palpation per rectum. Blood samples for progesterone analysis were obtained twice a week for 2 to 3 months following their spontaneous oestrous cycle, and all animals were rechecked for pregnancy at the end of the study. Progesterone analysis indicated that $33.9 \%$ of the total animals were having AOC: $18.9 \%$ with cessation of ovarian cyclicity, $9.4 \%$ with prolonged luteal phases (PLP), and 5.7\% short luteal phases. The highest incidence was observed in Brangus crosses $(62.5 \%)$, followed by Braford crosses (43.8\%), and Friesian crosses $(35.3 \%)$. In contrast, no AOC was observed in the local KKX breeds, and all of them were found to be pregnant at the end of the study. A significant difference $(p<0.05)$ in the incidence of AOC and its type was observed between Kedah-Kelantan crosses and the other breeds. Although not significant $(\mathrm{p}>0.05)$, Friesian crosses showed a higher percentage incidence of AOC than beef cows (40\% vs $36.4 \%$ ), with major types being PLP $(26.7 \%)$ in dairy and cessation of ovarian cycle $(27.3 \%)$ in beef cows. Compared with beef heifers, beef cows showed a higher percentage of AOC (36.4\% vs $28.6 \%)$ where again, cessation of cyclicity was the predominant abnormality. In conclusion, AOC reflected by abnormal endocrine pattern is a possible cause of reduction in fertility for dairy and beef cows beyond 90 days postpartum and heifers that fail to conceive.
\end{abstract}

Keyword: Conception failure, Cows, Heifers, Ovarian cycle, Progesterone, Prolonged postpartum period 